\title{
Review on Recent Approaches in Transdermal Drug Delivery System
}

\author{
Sirisha V and Sailaja $\mathrm{AK}^{*}$
}

Department of Pharmaceutics, RBVRR Women's College of Pharmacy, Barkatpura, Hyderabad, Telangana, India

*Corresponding author: Sailaja AK, Department of Pharmaceutics, RBVRR Women's College of Pharmacy Barkatpura, Hyderabad, Telangana, India, Tel: 040-27560365, E-mail: Shailaja1234@rediffmail.com

Citation: Sirisha V, Sailaja AK (2018) Review on Recent Approaches in Transdermal Drug Delivery System. J Nurs Patient Health Care 1(1): 103. doi: 10.15744/2767-9292.1.103

Received Date: May 17, 2018 Accepted Date: July 23, 2019 Published Date: July 25, 2019

\begin{abstract}
Transdermal drug delivery system has become a proven technology that offer significant clinical benefit over the dosage forms. Drugs with very short half-life, narrow therapeutic window, and poor bioavailability-transdermal drug system are convenient. Skin serves as site of drug application for local as well as systemic effects. There are wide varieties of drugs for which topical or transdermal is viable options. Skin penetration enhancement technique have been developed for such drugs, there are number of physical methods to increase drug delivery through the skin, many of which requires the usage of devices. This delivery has to full-fill some parameters such as high potency, better permeability through the skin and non-irritation for better compliance. Fick's-law of diffusion is the principle of drug kinetics. This review article provides the valuable information regarding the transdermal drug delivery system and highlights the detailed role of physical penetration and describes the kinetics of transdermal drug system and recent advance techniques in transdermal drug delivery system such as iontophoresis, sonophoresis, microneedles, electroporation.
\end{abstract}

Keywords: Transdermal; Drug Delivery

\section{Introduction}

In 1944 the term transdermal was coined by Merrimu Webster. In 1965, Stoughton first conceived of percutaneous absorption of drug substances. In 1970 Alza research (US) began first development of modern transdermal. The first transdermal system, transdermal scop, was approved by FDA in 1979 for prevention of nausea and vomiting. Transdermal drug delivery system overcomes the problems like hepatic first pass metabolism, improves patient compliance, decrease administration of frequency, and reduces gastro intestinal side effects, it offers controlled release of the drug into the patient, it enables a steady blood level profile, resulting in reducing systemic side effects.

\section{Transdermal Drug Delivery System}

Transdermal drug delivery systems are topically administered medicaments in the form of patches or semisolids that deliver drug for systemic effects at a predetermined and controlled rate [1].

Need

$\checkmark$ Drugs which undergoes extensive first pass metabolism can be formulate as Transdermal drug delivery system

$\Delta$ Drugs which are unstable in gastro intestinal environment can be formulate as Transdermal drug delivery system

$\checkmark$ If drug is having serious adverse effects, if action is required locally

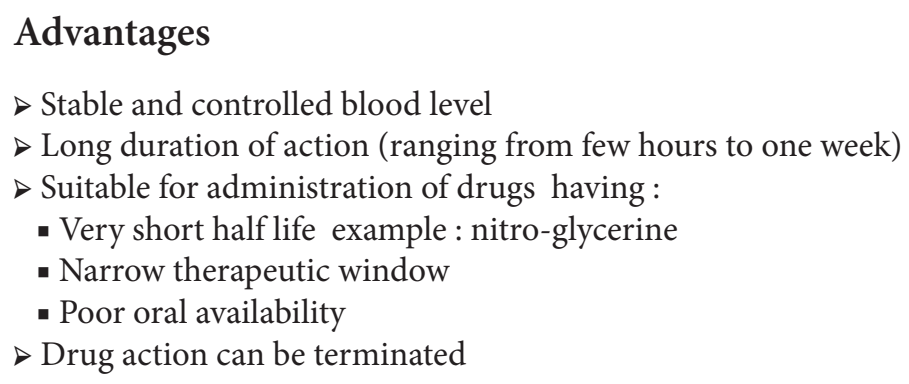


$\checkmark$ No interference with gastric and intestinal fluids

$\rightarrow$ Number of doses get reduces which improve patient compliance

$\checkmark$ Unwanted side effects get minimized

$\checkmark$ Self-medication is possible

$\rightarrow$ Brain targeting is possible by Transdermal drug delivery system [9].

\section{Disadvantages}

$\checkmark$ We cannot formulate all types of drugs as Transdermal drug delivery system

$>$ Drugs having affinity for both lipophilic and hydrophilic phase are used

$\rightarrow$ Drugs which are irritant to skin

$\Delta$ Difficult to administer large dose i.e. more than(10mg/day)

$\triangleright$ Drugs with very low or high partition coefficient fails to reach systemic circulation

$\rightarrow$ Ionic drugs create problem

$\checkmark$ Drugs undergoes protein binding in skin are not suitable [9].

\section{Structure of Skin and Barriers}

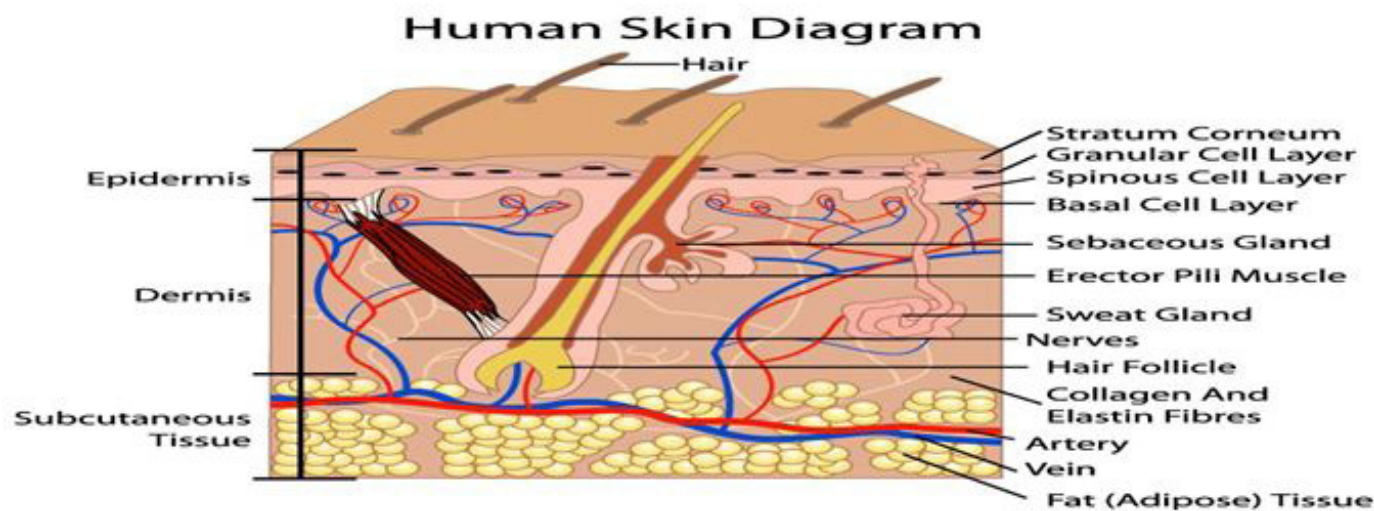

Figure 1:- Structure of skin and barriers

\section{The three basic layers of skin are as follows}

$\rightarrow$ Epidermis

$\rightarrow$ Dermis

$>$ Sub-cutaneous

Epidermis: It is the most superficial layer which is keratinized and composed of stratified squamous epithelial cells (Figure 1). Epidermis contains five layers i.e.

$\checkmark$ Stratum corneum

$\checkmark$ Stratum lucidum

$\checkmark$ Stratum granulosum

D Stratum spinosum

D Stratum basale

Stratum Corneum (Corneal Layer): Stratumcorneum is outer most layer of the skin which is actual physical barrier to pre cutaneous absorption. Stratum corneum is made up of 20-30 layers of dead, flat keratinocytes are continuously remove andreplaced by new cells from deeper layers [7]. Stratum corneum consists of lipid (5-15\%) including phospholipids, glycolsphingolipid, Cholesterol sulphate and neutrallipid,protein(75-85\%)mainly keratin. The strength of Stratum corneum is due to keratinocytes. Lipids make stratum corneum more lipophilic. Stratum corneum is dead epidermis, all other layers are living epidermis [5].

\section{Functions:}

$\checkmark$ This layer provides strength, elasticity to the skin

$\rightarrow$ Lipids impart water-repellent property

$\checkmark$ Since it is made up of dead cells, it protects the deeper layers from microbes and injury

$\triangleright$ It acts as a barrier for inward and outward movement of substance

Stratum Lucidum(Clear Layer): It is made up of 3-5 layers of flat, clear, dead keratinocytes, that are found only in areas such as finger tips. 


\section{Functions:}

$\nabla$ It protects the skin from harmful effects of UV

$\checkmark$ It also helps to reduce the friction between stratum corneum and stratum granulosum

Stratum Granulosum(Granular Layer): It is a thin middle layer composed of 3-5 layers of flat keratinocytes that are undergoing apoptosis i.e programmed cell death. This layer contain keratohylain that converts tonofilaments into keratin,keratin proteins and water proofing lipids are ipophilic and organized in this layer [3].

\section{Functions:}

$\checkmark$ Lipids prevent the entry and exit of water from skin

$\rightarrow$ This layer also prevents entry of foreign materials

Stratum Spinosum: It is 8-10 layers of polygonal keratinocytes tightly linked together by desmosomes [22].

\section{Functions:}

$\checkmark$ This layer provides strength and support to the skin and helps it to resist abrasion

Stratum Basale: It is the deepest layer of epidermis containing single layer of cuboidal keratinocytes. In this layer, few stem cells are present which divide continuously to form new cells which replace the older ones. In this tanofilaments are attached to desmosomes and hemidesmosomes [4].

Desmosomes: It helps to bind the cells of stratum basale with other and above epidermal layer.

Hemidesmosomes: such attachment helps the keratinocytes to bind with dermis.

Dermis: It is the deeper thicker layer of skin containing collagen fibres (provide strength), elastic fibres (provide flexibility) and reticular layer. Structurally it has two layers upper papillary layer and lower reticular layer

Upper papillary layer: It is composed of loose areolar connective tissue with thin collagen and fine elastic fibres. Some of dermal papillae contain capillary loops (blood vessels) that nourishes the epidermis [5].

Lower reticular layer: It is composed of dense connective tissue with fibroblasts, bundles of collagen fibres and coarse elastic fibres. These layer contains:

$\rightarrow$ Nerve ending

$\triangleright$ Blood vessels

$\triangleright$ Hair follicles

$\triangleright$ Glands

Beneath the dermis, there is a layer of subcutaneous tissue, containing a network of fat cells (adipocytes) and collagen fibres, called as Hypodermis. It protects from injuries by acting as a shock absorber [2].

\section{Mechanism of Transdermal Drug Delivery System:}

\section{Transepidermal Absorption:}

The mechanism of transdermal drug delivery system is diffusion of drug molecules from drug reservoir in the transdermal patch through the epidermal layers of the skin. The skin acts as a barrier and major obstruction is posed by stratum corneum, this is considered as rate-limiting membrane in transdermal drug delivery system. Permeation by trans epidermal route first involves partitioning into the stratum corneum, diffusion then takes place across the tissue. When permeating drug exits at stratum corneum it enters the wet cell mass of epidermis since the epidermis has no direct blood supply the drug is forced to diffuse across it, the epidermal cell membranes are tightly joined and there is no intercellular spaces for ions and polar non electrolyte molecule to diffusionally squeeze through. Thus permeation requires frequent crossings of cell membrane [10,7].

\section{Transfollicular Absorption:}

Follicular pores (opening on the skin where the hair shaft exists) of follicular glands are comparatively larger than the ipophili and eccrine sweat glands. These pores serve as the best root for percutaneous absorption. Partitioning of drug molecules into sebum followed by diffusion of drug molecules through the follicular pores up to the depth of epidermis, serves as mechanism of permeation by transfollicular route $[2,8]$.

\section{Kinetics of Transdermal Permeation:}

Transdermal permeation of a drug involves the following steps (Figure 2):

i. Sorption by stratum corneum 


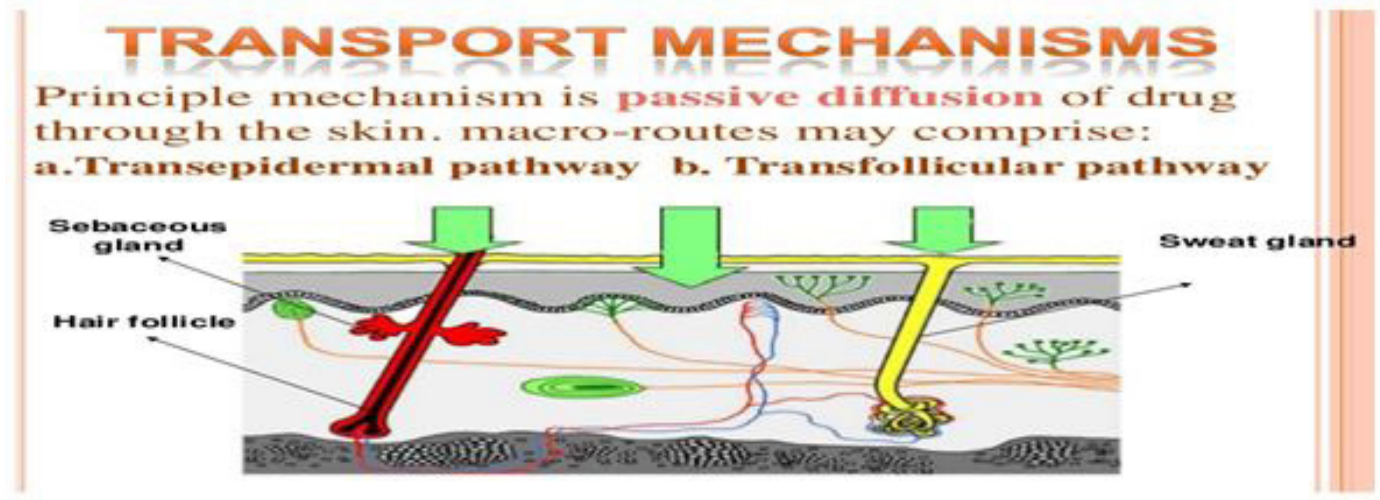

Figure 2:- Trans cellular transport mechanism

ii. Penetration of drug through viable epidermis

iii. Uptake of drug by capillary network in the dermal papillary layer

This permeation can be possible only if drug possess certain physic-chemical properties

The rate of permeation across the skin $\left(\frac{d q}{d t}\right)$ is given as

$$
\left(\frac{d q}{d t}\right)=\mathbf{P}_{\mathrm{s}}\left(\mathbf{C}_{\mathrm{d}}-\mathbf{C}_{\mathbf{r}}\right)-\cdots--(1)
$$

Where,

$\left(\frac{d q}{d t}\right)=$ rate of drug permeation

$\mathbf{P}_{\mathrm{s}}=$ Permeability coefficient of skin tissue towards penetrate (drug molecule)

$\mathrm{C}_{\mathrm{d}}=$ Concentration of drug in donor compartment (on the surface of stratum corneum)

$\mathbf{C}_{\mathbf{r}}=$ Concentration of drug in receptor compartment (amount of drug in systemic circulation)

The permeability co-efficient is given by:-Ps=KsxDss/hs

Where,

$\mathbf{K}_{\mathbf{s}}=$ Partion Co-efficient for interfacial partitioning of penetrant molecule from a transdermal therapeutic system on to the stratun corneum.

$\mathbf{D}_{\mathrm{ss}}=$ apparent diffusivity for study state diffusion of penetrate molecule through thickness of skin tissue.

Where,

$\mathbf{H}_{\mathrm{s}}=$ overall thickness of skin tissue.

As $\mathbf{K}_{\mathbf{s}}, \mathbf{D}_{\text {ss }} \mathbf{\&} \mathbf{h}_{\mathrm{s}}$ are constant under given conditions, the permeability co-efficient $\left(\mathbf{P}_{\mathbf{s}}\right)$ for a skin penetrant can be considered to be constant

From equation (1) it is clear that a constant rate of drug permeation can be obtained only when, $\mathbf{C}_{\mathbf{d}}>\mathbf{C}_{\mathbf{r}}$

Then equation (1) becomes

$\left(\frac{d q}{d t}\right)=\mathbf{P}_{s} \cdot \mathbf{C}_{\mathbf{d}}$

Keeping Cd constant, drug should be released from the transdermal device at a rate $\left(\mathrm{R}_{\mathrm{r}}\right)$ that is either constant (or) greater than the rate of skin uptake $\left(\mathbf{R}_{\mathbf{a}}\right)$

i.e $\quad \mathbf{R}_{\mathbf{r}}>>\mathbf{R}_{\mathbf{a}}$

Since $\mathrm{Rr}$ is greater than $\mathrm{Ra}$, Cd (drug con of skin) is maintained at a level equal (Or) greater than the (Cs) equilibrium solubility of drug in stratum corneum.

i.e $\mathbf{C}_{\mathbf{d}}>\mathrm{C}_{\mathrm{s}}$

Therefore a maximum rate of skin permeation $\left(\frac{d q}{d t}\right)_{\max }$ is obtained by equation $\left(\frac{d q}{d t}\right)_{\max }=\mathbf{P}_{\mathrm{s}} \cdot \mathbf{C}_{\mathrm{s}}$

From the above equation it can be seen that maximum rate of skin permeation depends on skin permeability co-efficient $\left(\mathbf{P}_{\mathbf{s}}\right)$ and its equilibrium solubility in stratum conium $\left(\mathbf{C}_{\mathbf{s}}\right)$. 
Therefore Penetration of drug through stratum conium is Rate - limiting step. ${ }^{1}$

Transdermal DDS follows - first order kinetics.

\section{Basic - Components of Transdermal Drug - Delivery System:}

1. Polymer Matrix (Or) Matrices

2. Drug

3. Permeation enhancers

4. Other Excipients

Polymer Matrix: Polymer controls the release of the drug from device. The following criteria should be satisfied for a polymer to be used in transdermal system.

(i) Molecular weight, glass transition temperature and chemical functionality of polymer should be such that specific drug diffuses properly and get released through it.

(ii) Polymer should be stable, non-reactive with drug, easily manufactured and fabricated into the desired properly and inexpensive.

(iii) Polymer and its degradation products must be non-toxic.

(iv) The mechanical properties of polymer should not deteriorate excessively when large amount of active agent are incorporated into it [13].

\section{Possible Useful Polymers for Transdermal Devices are}

$\checkmark$ Natural Polymers: Cellulose derivatives, Zein, Gelatin, Shellac, Waxes, Proteins, gums and their derivatives, Natural rubber, Starch etc.,

$\checkmark$ Synthetic Elastomers: Poly butadiene, Hydrin rubber, Poly siloxane, silicone rubber, nitrile, Acrylonitrile, Butyl rubber, styrene butadiene rubber, Neoprene etc.,

D Synthetic Polymers: Poly vinyl alcohol, Poly vinyl chloride, Polyethylene, Polypropylene, Polyacrylate, Polyamide, Polyurea, Poly vinyl pyrrolidone, Poly methyl methacrylate, Epoxy etc.,

Drug: For a successful developing transdermal DDS, the drug should be chosen with great care. The following are the some of the desirable properties of drug for transdermal delivery [14].

\section{Physiochemical Properties:}

$\checkmark$ The drug should have a molecular weight less than approximately 1000 daltons

$\checkmark$ The drug should have affinity for both lipophilic and hydrophilic phases [18].

\section{Biological Properties:}

$\checkmark$ The drug should be potent with a daily dose of a few mg/day

$\rightarrow$ The half-life $(\mathrm{t} 1 / 2)$ of the drug should be short

$\checkmark$ The drug must not induce a cutaneous irritant (or) allergic response

$\checkmark$ Drug which degrade in GI tract (or) are inactivated by hepatic first-pass effect are suitable candidates for transdermal delivery

$\checkmark$ Tolerance to the drug must not develop under the near zero-order release profile of transdermal delivery [15].

Permeation Enhaners: (or) penetration enhances

Permeation enhances are also known as accelerants (or) sorption promoters, these are the compounds that promote the permeability of the stratum corneum to the flux (J) of drug molecules in order to attain higher therapeutic levels. ${ }^{15}$

Flux (process of flowing) of the drug melecules across the skin is given by equation,

$\mathbf{J}=\mathbf{D}\left(\frac{d c}{d x}\right)$

Where,

$\mathrm{D}=$ diffusion co-efficient

$\mathbf{C}=$ concentration of diffusing species

$\mathbf{X}=$ spatial co-ordinate (how $\mathrm{mol}$ is arranged)

\section{Thus enhancement of flux across membrane reduces to considerations of:}

$\checkmark$ Thermodynamics (lattice energies, distribution co-efficient)

$\checkmark$ Molecular size and shape

$\checkmark$ Reducing the energy required to make a molecular hole in the membrane [16]. 


\section{Mechanisms of Permeation Enhancers:}

$\triangle$ By disrupting the structure of stratum corneum lipids

$\checkmark$ By interacting with intercellular proteins

$\checkmark$ By improving drug partitioning, Co-enhancer (or) solvent into the stratum corneum

$\checkmark$ Dissolution of drug in its vehicle

$\checkmark$ Diffusion of drug from vehicle to surface of skin [15]

\section{Ideal Characteristics of Enhancers:}

$\checkmark$ The effect of enhancer on the skin should be reversible, and it should not damage the viable cells

$\nabla$ It should show their effect rapidly, with predictable and reproducible duration of effect

$\checkmark$ It should have unidirectional enhancing effect, allowing the drug molecule to pass through the skin shield preventing the loss of endogenous materials like body fluids, electrolytes etc.,

$\checkmark$ It should be pharmacologically riel, non-toxic, non-allergic, non- irritating

$\checkmark$ It should be compatible with drug and other additives

$\nabla$ It should be economical [12].

\section{Commonly employed permeation enhances are listed below:}

Solvents: These compounds increase penetration possibly by swelling the polar pathway (or) by fluidizing lipids.

Examples: water alcohols - methanol and ethanol, alkyl methyl sulfoxides-dimethyl sulfoxide, alkyl homologs of methyl sulfoxide, dimethyl acetamide and dimethyl formamide pyrrolidones-2 pyrrolidone, N-methyl, laurocapram (azone), Miscellaneous solvents - propylene glycol, glycerol, silicone fluids, isopropyl palmate.

Surfactants: These compounds are proposed to enhance polar pathway transport, especially of hydrophilic drugs the ability of a surfactant to attend penetration is a function of polar head group and hydrocarbon chain length. These compounds are, however, skin irritants. The 3 major types of surfactants are:

I. Anionic Surfactants: - can perpetrate and interact strongly with skin. Ex: - SLS, dioctyl sulphosuccinate, decodecyl methyl sulphoxide etc.

II. Cationic Surfactants: - These surfactants penetrate into the skin and extract lipids from stratum corneum. Hence they cause more skin irritation than anionic surfactants, hence they are not employed as permeation enhances. Ex: - Dodecyltrimethyl ammonium bromide

III. Non-ionic Surfactants: They are widely used Ex: plutonic F127, Plutonic F68

IV. Bile Salts: sodium taurocholate, sodium deoxycholate, sodium tauroglycocholate.

V. Binary Systems: These systems apparently open up the heterogeneous multilaminate pathway as well as continuous pathway Ex: Propylene glycol - oleicaI, U-but are dial - linoleic a

\section{Miscellaneous agents:}

Urea: - It is a catalytic agent that functions by increasing the fluidity of intercellular bilayers, hence hydrating the stratum conium $\checkmark$ Phosphatide choline: It diffuses into the stratum conium, Extracts intercellular lipids and hence promotes the penetration of drug into the skin

$\rightarrow$ Calcium thioglycolate

$\checkmark \mathrm{N}, \mathrm{N}$ - dimethyl - m - toluidine [18].

Other Excipients:

a) Adhesives: Pressure sensitive adhesive is a material that helps to adhere transdermal devices to the skin over long periods of time. These adhesives can be placed on the face of device known as face adhesive system (or) peripherally at the back of device known as peripheral adhesive system [9].

Widely used pressure sensitive adhesives include polyisobutylenes, acrylics and silicones.

Backing membranes: These are flexible and they provide a good bond to drug reservoir, and also accept printing it is impermeable substance that protects the product during use on the skin.

Examples of baking membranes: Metallic plastic laminate, flexable adhesive foam pad with occlusive base plate, plastic backing with absorbent pad and occlusive base plate (aluminium foil) disc [8].

\section{Transdermal DDS Can be classified into 2 major categories:}

\section{A. RATE PROGRAMMED TRANSDEMRAL DRUG DELIVERY SYSTEM. \\ B. PHYSICAL STMULI-ACTIVATED DRUG DELIVERY SYSTEM.}




\section{Rate-Programmed Transdermal DDS/Transdermal Patches:}

I. Membrane permeation - controlled systems (Drug in reservoirs)

II. Adhesive dispersion - type systems (Drug in adhesive matrix)

III. Matrix diffusion - controlled system (Drug in matrix)

IV. Micro reservoir type (or) micro sealed dissolution controlled system

\section{Several Features are Common to all Systems Transdermal DDS and Includes}

Release liner: - a protective cover that is peeled away before applying the path.

Impermeable backing layer: This layer prevents Transdermal drug delivery system from water, dust, microorgansms.

Rate - Controlling Polymer membrane: It controls the release of drug from reservoir.

Pressure Sensitive Adhesive: There are three critical considerations in the selection of a particular system:

a. Adhesion to skin

b. Compatibility with skin

c. Physical (or) chemical stability of total formulation and Components.

\section{A. Rate Programmed Transdemral Drug Delivery System:}

\section{Membrane - Permeation - Controlled Systems:}

In this type of system, the drug reservoir is totally encapsulated in a shallow compartment mound from a drug - impermeable metallic plastic laminate and a rete controlling polymeric membrane which may be micro porous (or) non-porous (Figure 3). Ex: Ethylene-Vinyl acetate co-polymers, silicones, high density polyethylene, polyesterelastomers and polyacrylonitride. The drug molecules are permitted to release only through the rate - controlling membrane. In the drug reservoir compartment drug solids are either dispersed in a solid polymer matrix (or) suspended in a viscous liquid medium. A definite requirement for a reservoir system is that it should permit zero-order release of the drug over the delivery period. A thin layer of adhesive polymer is placed below the membrane to secure the TDDs over the skin. The polymer is designed to be compatible with drug and hypoallergenic [1].

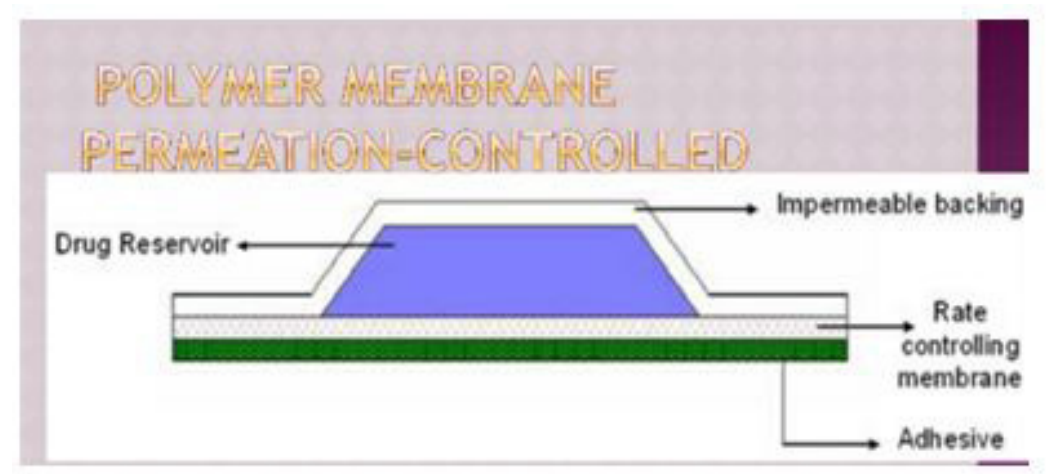

Figure 3:- Membrane permeation controlled system

The intrinsic rate of drug release from this type of drug delivery system is defined by

$\left(\frac{d q}{d t}\right)=\mathbf{c}_{\mathrm{r}} / \mathbf{1} / \mathbf{P}_{\mathrm{m}}+\mathbf{1} / \mathbf{P}_{\mathrm{a}}$

Where,

$\mathrm{C}_{\mathrm{r}}=$ Concentration of drug in the drug reservoir.

$\mathbf{P}_{\mathbf{a}}=$ Permeation Co-efficient of adhesive layer.

$\mathbf{P}_{\mathbf{m}}=$ Permeation Co-efficient of rate controlling membrane.

For any micro porous rate - controlling membrane, $\mathrm{Pm}$ approximately represents the sum of permeability co-efficient across the pores and polymeric material. $\mathbf{P}_{\mathbf{a}}$ and $\mathbf{P}_{\mathbf{m}}$ may be separately defined as $\mathbf{P}_{\mathbf{a}}$

$\mathrm{P}_{\mathrm{a}}=\mathrm{K}_{\mathrm{a} / \mathrm{m}} \cdot \mathbf{D}_{\mathrm{a}} / \mathbf{h}_{\mathrm{a}}$

$\mathrm{P}_{\mathrm{m}}=\mathrm{K}_{\mathrm{m} / \mathrm{r}} \cdot \mathrm{D}_{\mathrm{m}} / \mathbf{h m}$

Where,

$\mathbf{D}_{\mathbf{a}}=$ Diffusion Co-efficient of an derive layer

$\mathbf{D}_{\mathrm{m}}=$ Diffusion Co-efficient of rate - controlling membrane 
$\mathbf{K}_{\mathbf{a} / \mathbf{m}}=$ Partition Co-efficient for interfacial partitioning of drug from rate controlling membrane to adhesive layer

$\mathbf{K}_{\mathbf{m} / \mathbf{r}}=$ Partition Co-efficient for interfacial partitioning of drug from reservoir to rate controlling membrane

$\mathbf{h}_{\mathrm{m}}=$ Thickness of rate $=$ Controlling membrane .

$\mathbf{H}_{\mathbf{a}}=$ Thickness of adhesive layer.

Advantage: This type of TDDS provides constant release pattern of the drug.

Disadvantage: Accidental breakage of the device may cause Dose Dumping (or) sudden release of entire Drug from Drug reservoir.

\section{Examples of Membrane - Permeation Controlled Systems}

$\rightarrow$ Nitroglycerine releasing transdermal system for 1day in Angina Pectoris.

$\checkmark$ Scopolamine releasing transdermal system Every 72 Hours in Motion Sickness

$\checkmark$ Clonidine releasing transdermal system for 7 Days Therapy of Hypertension

$\checkmark$ Estradiol releasing transdermal system 3-4 Days for Menopausal Syndrome

\section{Adhesive Dispersion - Type Systems:}

This is a Simplified form of membrane Permeation-Controlled Systems (Figure 4). In this system, drug and other selected excipients are directly incorporated into the adhesive solution. They are then mixed and casted as thin films and finally the solvent is evaporated by drying the film. The drug reservoir (film) is the then sandwiched between the banking laminate and rate - controlling adhesive polymer membrane [6].

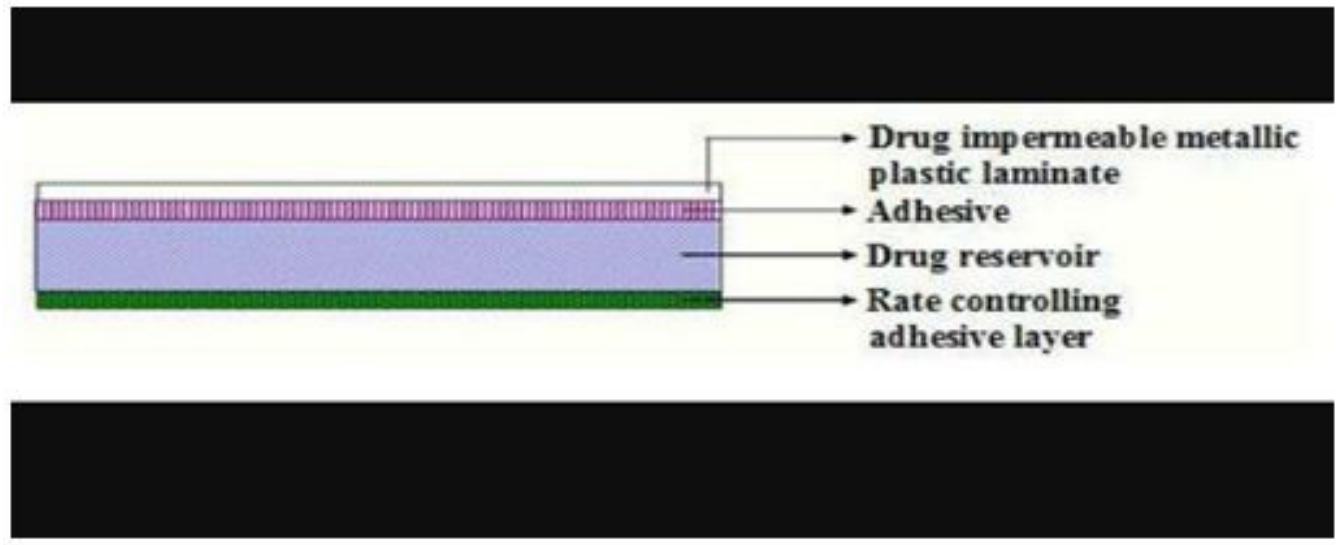

Figure 4:- Adhesive Dispersion - Type Systems

The rate of drug release from this system is given by, $\left(\frac{d q}{d t}\right)=\mathbf{C}_{\mathbf{r}} \cdot \mathbf{K}_{\mathbf{a} / \mathbf{r}} \cdot \mathbf{D}_{\mathbf{a}} / \mathbf{h}_{\mathbf{a}}$

Where $\mathbf{K}_{\mathrm{a} / \mathbf{r}}=$ Partition co-efficient for interfacial partitioning of drug from reservoir layer to adhesive layer.

Examples: Iso sorbide dinitrate - releasing TDDS - $24 \mathrm{hr}$, Used in Angina Pectoris

Verapamil - releasing TDDS - 24 hrs, used in Hypertension.

This system have certain disadvantages: Physiochemical characteristics of the drug and adhesive system may provide very different release rates for hydrophilic and hydrophobic drugs. Incorporation of other excipients, such as skin permeation enhancers, into drug in adhesive system may alter drug release rates and adhesive properties.

III. Matrix Diffusion-Controlled Systems: In this the drug reservoir is prepared by homogeneously dispersing drug particles in a hydrophilic (or) lipophilic polymer matrix (Figure 5). The resulting polymer matrix is then moulded into discs with defined surface area and controlled thickness. The medicated disc is then moulded onto an occlusive base plate in a compartment made up of a drug impermeable backing. Finally adhesive polymer is spread along the circumference of the film [7].

Rate of drug release in this system is given by the equation $\left(\frac{d q}{d t}\right)=\mathbf{A C}_{\mathbf{p}} \mathbf{D}_{\mathbf{p}} / \mathbf{2 t}$

Where,

$\mathbf{A}=$ Initial drug loading dose dispersed in polymer matrix

$\mathbf{C}_{\mathbf{p}}=$ Solubility of drug in Polymer

$\mathbf{D}_{\mathbf{p}}^{\mathbf{p}}=$ Diffusivity of drug in Polymer since $\mathbf{C}_{\mathbf{p}}$ is equal to $\mathbf{C}_{\mathbf{r}}$

Where, 


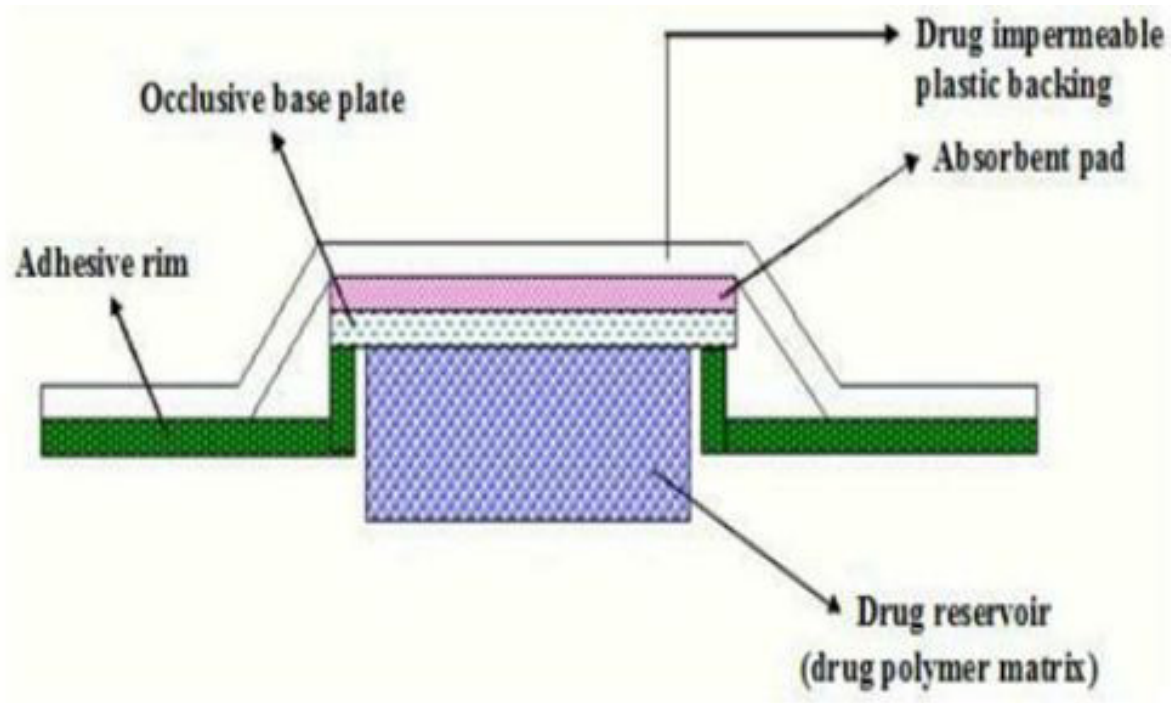

Figure 5:- Matrix diffusion controlled Systems

Advantage: Due to presence of polymer with the drug reservoir, dose dumping may be avoided.

Examples: Nitro-glycerine releasing transdermal therapeutic system at a daily dose of $0.5 \mathrm{~g} / \mathrm{cm} 2$ for angina pectoris.

IV. Micro-reservoir type system: This drug delivery system is a combination of reservoir and matrix- dispersion system (Figure 6). The drug reservoir is formed by first suspending the drug in an aqueous solution of water soluble polymer like PEG and then dispersing the solution homogeneously in a ipophilic polymer to form thousands of unleachable, microscopic spheres of drug reservoirs. The thermodynamically unstable dispersion is stabilized quickly by immediately cross linking the polymer [17].

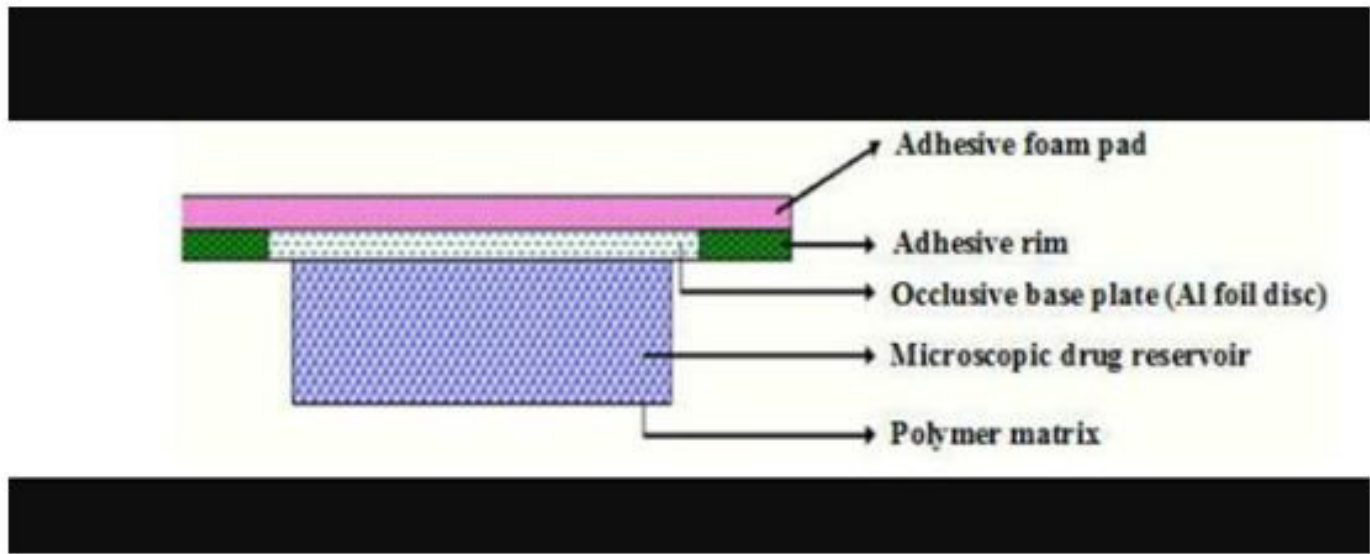

Figure 6:- Micro reservoir type Systems

Advantages: No dose dumping

\section{Physical Stmuli-Activated Drug Delivery System}

\section{Electrically based systems:}

- Iontophoresis

- Electroporation

- Sonophoresis

- Microneedles

Iontophoresis: Ionotophoresis is the process of enhancing the permeation of topically applied therapeutic agents (Figure 7). The principle of iontophoresis technique is based on that the like charges repels each other and opposite charge attract. Thus during iontophoresis, if delivery of positively charged drug, the charged drug is dissolved in the electrolyte surroundings the electrode of similar polarity i.e. Anode. An application of electromotive force, the drug is repelled and moves across the stratum corneum towards the cathode, which is placed elsewhere on the body. The drug permeation is caused by any 1 (or) combination of the following mechanisms [21]. 
$\triangle$ Electro - repulsion (for Similar charged solutes)

$\checkmark$ Electro - Osmosis (for uncharged solutes)

$\triangleright$ Electro - Perturbation (for both charged and uncharged solute)
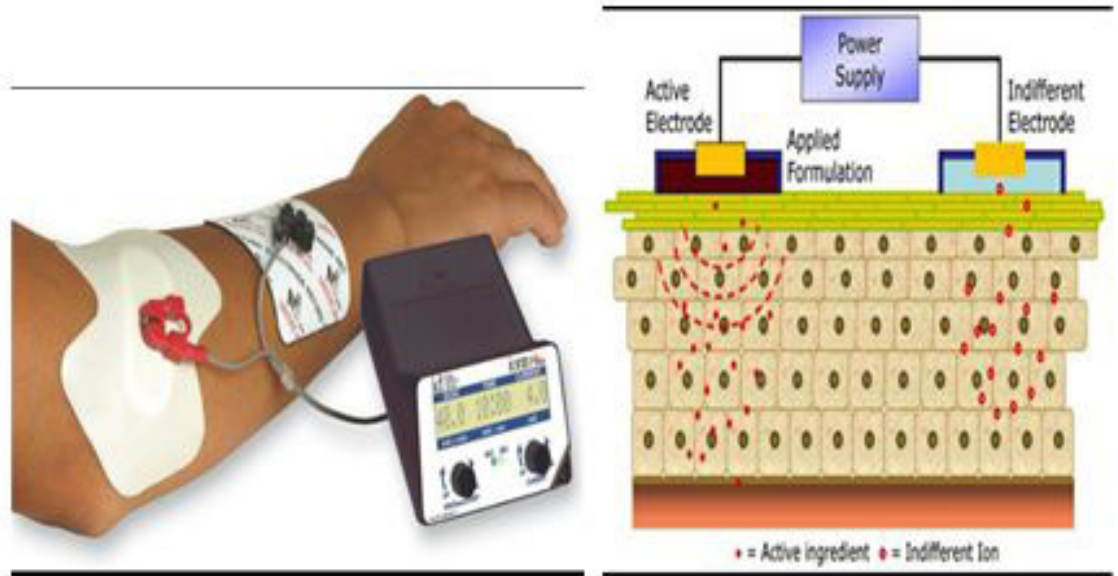

Figure 7:- Iontophoresis for drug permeation through skin

Electroporation: It is the phenomenon in which cell membrane permeability to ions and macromolecules is increasing by exposing the cell to short high electric field pulses. The mechanism for electroporation by 2 pathways, through pores formed in multiple lipid bilayer connecting corneocytes, through appendage cells. The efficacy of transport of drug depends on the electrical parameters and physiochemical properties or drugs. In electroporation the transdermal delivery of drug molecule is increased by application of a high voltage (100 volts) in form of direct current (DC) [13].

Sonophoresis: Here ultrasonic energy enhances the transdermal delivery of solutes (Figure 8). Drug is mixed with a coupling agent usually gel, cream (or) ointment. The coupling agent acts as a medium for the transfer of phonophoretic energy from the device to the skin. Sonophoresis causes disruption of stratum corneum by cavitations, micro screening and heat sensitization. This involves rupturing the lipids present in stratum cornea, which allows medication to permeate via biological barrier. It uses low frequency ultrasound (55KHZ) for an average duration of $15 \mathrm{sec}$ to enhance skin permeability [15].

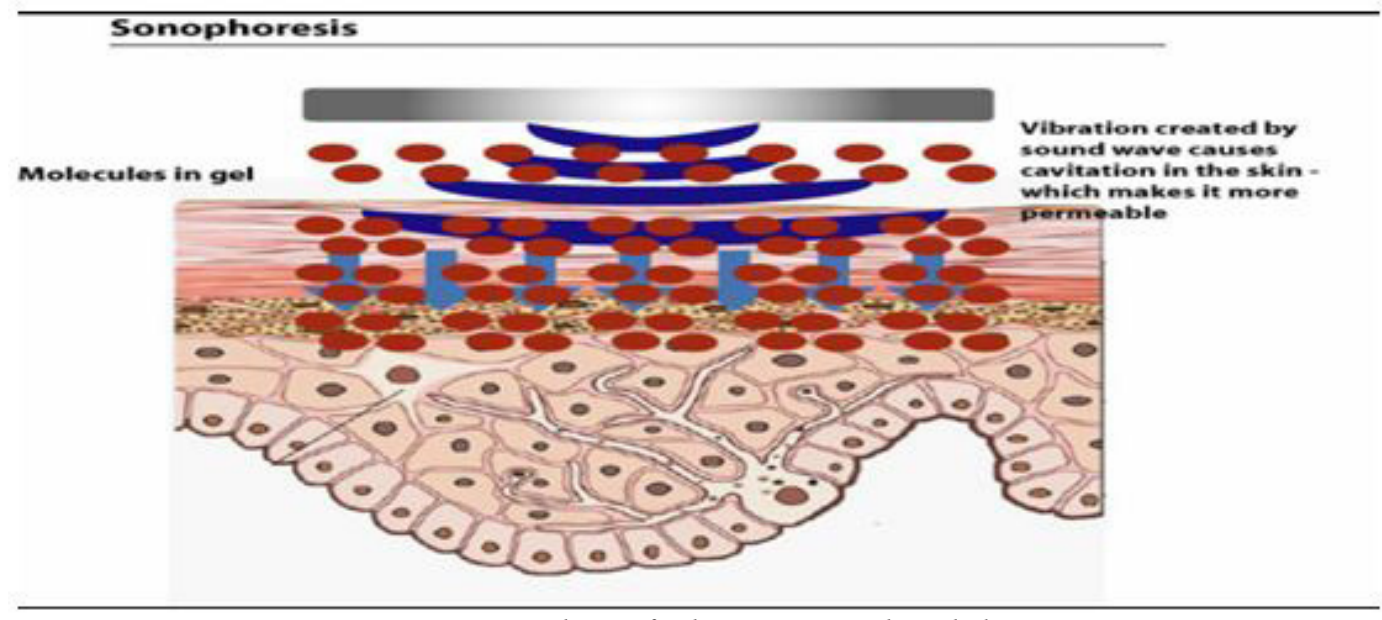

Figure 8:- Sonophoresis for drug permeation through skin

Magnetophoresis: This method involves the application of a magnetic field which acts as an external driving force to increase diffusion of diamagnetic solute across the skin. Skin exposure to magnetic field might also induce structural alternations, that could contribute to an increase in permeability magnetoliposome's consist of magnetic nanoparticles wrapped by a phospholipids bilayer which can be successfully applied for drug delivery system, magnetic resonance imaging markers for cancer diagnosis and thermal cancer therapy [13].

Micro needles: This technique is also called as microscoping projection, as the name indicates the device contains microprojections which are arranged in array (Figure 9). The microneedles of length 50 to $110 \mu \mathrm{m}$ will penetrate the stratum corneum and epidermis to deliver the drug from the reservoir, the reservoir may contain drug, solution of drug, gel (or) solid particulates. The micro needle array is attached to the electronic device and placed in contact with the skin to facilitate the formation of micro channels. Treatment duration takes less than a second, with a feedback mechanism incorporated with the electronic control providing a signal where micro-channels have been created. The drug patch is then placed on treated area [12]. 


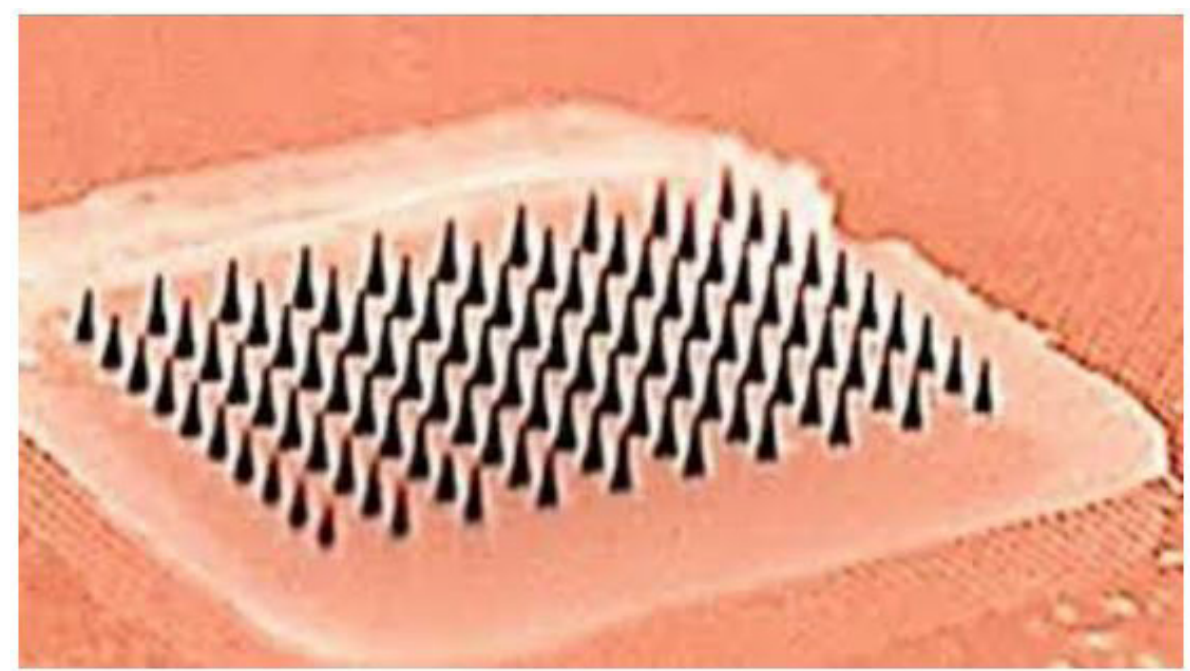

Figure 9:- Micro scoping projection for drug permeation through skin

\section{References}

1. Jain NK (2001) Controlled and novel drug delivery. (1 $1^{\text {st }}$ Edn.) CBS Publisher and Distributors, New Delhi, India 2001: 100-29.

2. Jalwal P, Jangra A, Dhaiya L, Sangwan Y, Saroha R (2010) A review on transdermal patches. Pharm Res J 3: 139-49.

3. Bharadwaj S, Garg VK, Sharma PK, Bansal M, Kumar N (2011) Recent advances in transdermal drug delivery system. Int J Pharm Tech Res 2: 68-77.

4. Kumar A, Pullankandam N, Prabhu SL, Gopal V (2010) Transdermal drug delivery system: an overview. Int J Pharm Sci Review Res 3: 49-54.

5. Divya A, Rao MK, Gnanprakash K, Sowjanya A, Vidyasagar N, et al. (2012) A review on current scenario of transdermal drug delivery system. Int J Res Pharm Sci 3: 494-502.

6. Robinson JR, Lee VH (2005) Controlled drug delivery fundamentals and applications. ( $2^{\text {nd }}$ Edn.)New York: 523-36.

7. Wilson R, Waugh A, Grant A (2001) Anatomy and physiology in health and illness. (9 $9^{\text {th }}$ Edn.) 2001: 363-6.

8. Kumar D, Sharma N, Rana AC, Agarwal G, Bhat ZA (2011) A review: transdermal drug delivery system: a tools for novel drug delivery sestem. Int J Drug Dev Res 3: 70-84.

9. Chein YW (1992) Novel drug delivery systems, Drugs and Pharmaceutical Sciences. (2 ${ }^{\text {nd }}$ Edn.) Marcel Dekker ,New York: 797.

10. Arunachalam A (2010) Transdermal Drug Delivery System : A Review. Cur Pharma Res 1:70-81.

11. Vishwakarma SK, Niranjan SK, Irchhaiya R, Kumar N, Akhtar AA Novel transdermal drug delivery system. Int J res pharm 2: 39-44.

12. Shingade GM, Aamer Q, Sabale PM, Gramprohit ND, Gadhave MV, et al. (2012) Review on: recent trend on transdermal drug delivery system. J Drug Delivery Therap 2: 66-75.

13. Rastogi V, Yadav P (2012) Transdermal drug delivery system: An overview. Asian Journal of Pharmaceutics 6: 161-70.

14. Mehta RS, Patel DM, Bhatt KK, Shankar MB (2005) UV and visible spectrophotometric analysis of pioglitazone hydrochloride in bulk and tablets. IJPS : 87-9.

15. Anisree GS (2012) A research on formulation and evaluation of Transdermal Drug Delivery System. J pharm sci res 4: 1939-49.

16. Kakar S, Singh R, Semwal A (2014) Transdermal Drug Delivery As A Boon. Asian Pacific J Health Sci 1: 13-25.

17. Kumar R., Philip A. (2007) Modified transdermal technologies: breaking of the barrier of drug permeation via the skin. Trop J Pharm Res 6: 633-44.

18. Shaila L, Pandey S, Udupa N (2006) Design and evaluation of matrix type membrane controlled Transdermal drug delivery system of nicotin suitable for use in smoking cessation. IJPS 68: 179-84.

19. Rastogi V, Yadav P (2012) Transdermal drug delivery system: An overview. AJPS 6: 161-70.

20. Soni S, Dixit VK (1992) Transdermal delivery system. Indian Drugs 29: 466-7.

21. Panchagnula R (1997) Transdermal delivery of drugs. Indian J Pharmacol 29: 140-56.

22. Tortara GS, Grabowski SK (2000) Principles of Anatomy and Physiology, ( th $^{\text {th }}$ Edn.) 140-94. 


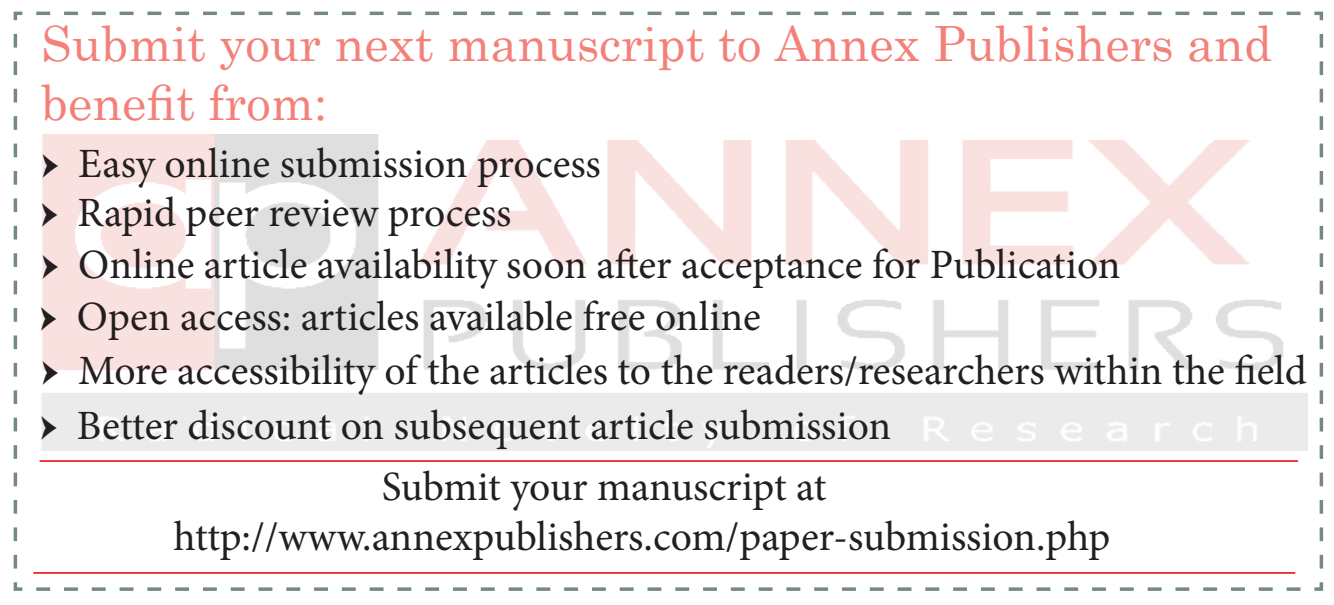

